\title{
NUMERICAL SOLUTION OF LINEAR DIFFERENTIAL EQUATIONS BY WALSH POLYNOMIALS APPROACH
}

\author{
GYÖRGY GÁT*1, and RODOLFO TOLEDO ${ }^{2}$ \\ ${ }^{1}$ Institute of Mathematics, University of Debrecen, H-4002 Debrecen, Pf. 400, Hungary \\ e-mail: gat.gyorgy@science.unideb.hu \\ ${ }^{2}$ Institute of Mathematics and Computer Science, University of Nyíregyháza, H-4400 \\ Nyíregyháza, Pf. 166, Hungary \\ e-mail: toledo.rodolfo@nye.hu
}

Communicated by Sz. Révész

(Received August 9, 2019; accepted May 25, 2020)

\begin{abstract}
In 1975 C. F. Chen and C. H. Hsiao established a new procedure to solve initial value problems of systems of linear differential equations with constant coefficients by Walsh polynomials approach. However, they did not deal with the analysis of the proposed numerical solution. In a previous article we study this procedure in case of one equation with the techniques that the theory of dyadic harmonic analysis provides us. In this paper we extend these results through the introduction of a new procedure to solve initial value problems of differential equations with not necessarily constant coefficients.
\end{abstract}

\section{Introduction}

A system formed by Walsh functions is an orthonormal system which takes only values 1 and -1 . This property, which is why the Walsh system was considered to be an "artificial" orthonormal system by many mathematicians in 1923, the year of its introduction (see [21]), offers a wide range of applications in the world of digital technology. Indeed, the Walsh functions have a great advantage with respect to the classic trigonometric functions

2010 Mathematics Subject Classification. Primary 42C10; Secondary 65L05.

Key words and phrases. Fourier analysis; Walsh-Paley system; numerical solution of linear differential equations; dyadically circulant matrices; triangular functions; uniform convergence.

* Corresponding author 
in the sense that computers can be very effective to determine the precise value of any Walsh function at any point.

In the 1970s the potential of piecewise constant orthogonal systems for signal characterization became evident. It was the reason why several researchers began to study intensively the application of Walsh functions in communication and signal processing (see, among others, $[1,12,14,16,17]$ ). In [8] Corrington developed a method to solve $n$th order linear differential equations using previously prepared huge tables of the Walsh-Fourier coefficients of weighted indefinite integrals of Walsh functions.

Corrington derived $n$ different tables for solving an $n$th order differential equation. In 1975 C. F. Chen and C. H. Hsiao improved the method of Corrington where only one table is needed (see [2]). Moreover, this table contains elements easy to compute (see the matrices in (7) and (8)). This was possible by considering the equivalent system of linear differential equations of first-order. The new approach is much simpler and more suitable for digital computation. Although we must also say that the method of Chen and Hsiao is only suitable for solving linear differential equations with constant coefficients having initial conditions at $x=0$.

In 1975 Chen and Hsiao wrote several papers in which they show the performance of their procedure in applications. Papers [6] and [4] present a new approach to the optimal problem by using Walsh functions. In [5] they dealt with the application of Walsh functions to the time-domain-synthesis problem. Paper [3] establishes a clear procedure for the variational problem solution via the Walsh functions technique. On the basis of this method it was also possible to develop a technique for the analysis of time-invariant linear delay-differential equations by the Walsh polynomials approximation (see [7]) and also another for solving first-order partial differential equations by double Walsh series approximation (see [19]).

The basic idea of the method of Chen and Hsiao is to avoid differentiation considering the equivalent integral equations instead the original differential equations, because the Walsh functions are not differentiable. They discretize these integral equations substituting all functions in them, even the integral functions, by the partial sums of Walsh series of these functions. Every component of the exact solution is also substituted by an unknown Walsh polynomial. The aim is to find the coefficients of these polynomials which are obtained after solving a linear system.

However, Chen and Hsiao did not deal with the extensive analysis of the proposed numerical solution. We mean that they did not determine if the linear system is solvable or not, neither did they deal with the estimation of errors. On the other hand, to obtain great accuracy for the numeric solution we need to solve a linear system involving a very large number of variables and equations. The construction of Walsh polynomials from their coefficients also requires time. It is possible to design a procedure to obtain directly the values of the Walsh polynomials? What is the largest class of constant terms where the method works? 
In [10] and [11] we started with the analysis of these issues considering the simplest case, i.e. studying the approximation by Walsh polynomials of the solution of one linear differential equation with constant coefficient having an initial condition at $x=0$. In other words, we studied the initial value problem

$$
\begin{aligned}
y^{\prime}+a y & =q(x), \\
y(0) & =\eta,
\end{aligned}
$$

where $a, \eta \in \mathbf{R}$. The Walsh functions are defined on the interval [0,1[, so the solution $y$ should be found in this interval. The continuity of the constant term $q(x)$ on the interval $[0,1$ [ ensures the existence and the uniqueness of the solution, but the continuity on the closed interval $[0,1]$ is not needed. It is enough to suppose that the function $q$ is integrable, i.e.

$$
\int_{0}^{1}|q(x)| d x<\infty,
$$

since the method requires the computation of Walsh-Fourier coefficients.

For every positive integer $n$ the procedure of Chen and Hsiao gives us an unique Walsh polynomial of the form

$$
\bar{y}_{n}(x)=\sum_{k=0}^{2^{n}-1} c_{k} w_{k}(x),
$$

where $w_{k}$ is the $k$ th Walsh function ordered in Paley's sense. In [11] we proved that, with very rare exceptions, $\bar{y}_{n}$ can always be constructed. Only if $2^{n+1}=-a$ we can not solve the linear system from which we obtain the coefficients of $\bar{y}_{n}$, and this only happens at most for one value of $n$ in a given differential equation. It was also proved that $\bar{y}_{n}$ converges uniformly to the exact solution $y$ if $n$ tends to infinity.

The Walsh polynomial $\bar{y}_{n}$ is a piecewise constant function on intervals of length $\frac{1}{2^{n}}$. To obtain the coefficients of $\bar{y}_{n}$ we solve a linear system of dimension $2^{n} \times 2^{n}$. This means that for a good accuracy in many cases we most solve a very large linear system. In [20] the block structure of the coefficient matrix is used to accelerate the computations, representing it by Quantum multiple-valued decision diagrams. However, in [11] we developed a multistep algorithm to obtain directly the values of $\bar{y}_{n}$. This allow us to do a really quick and efficient computation of the numerical solution.

In this paper we establish a similar method for solving numerically differential equations with not constant coefficient. Our aim is to design a new procedure to approach by Walsh polynomials the solution of a general linear 
differential equation of first-order having an initial condition at $x=0$. The basic idea is the same, but the the complexity of the procedure increases and the analysis of the proposed numerical solution requires a solid mathematical background. However, we obtain excellent results which are compatible with those in [11]. We summarize these results and describe our new method in the next section.

Sections 3, 4 and 5 contain the necessary mathematical concepts and statements for the precise analysis of the method which is implemented in all its details in Section 6. In this section we also deal with the solvability of the linear system, in other words, with the existence of the proposed numerical solution. It turns out that there exists an unique numerical solution, except for finite numbers of $n$. In Section 7 we prove that the numerical solution converges uniformly to the exact solution of the initial value problem, as we state in Theorem 1.

In Section 8 we propose a multistep algorithm to speed up the computations. In this way we directly obtain the values of the numerical solution without needing to solve the linear systems and generate Walsh polynomials. Algorithms of this kind are used frequently for solving differential equations numerically. Among them we would like to mention the method developed by Lukomskii and Terekhin in [15] to approximate the derivative of the solution by step functions for solving first order linear Cauchy problem with continuous coefficient and free term on the close interval $[0,1]$. Our multistep algorithm only involves the integral means of these functions, so for us it is sufficient to suppose the continuity of them on the interval $[0,1[$.

Our method is illustrated in Section 10 through three examples. The first one solves a Cauchy problem with continuous coefficient and free term on the close interval $[0,1]$. The second example shows us the uniform convergence of the numerical solution in case of integrable coefficient and free term which are only continuous on the interval [0,1[. The third example illustrates how the multistep algorithm also works in case of not integrable functions. We discuss in detail the last case in Section 9.

\section{Notation and main results}

Consider the following initial value problem

$$
\begin{aligned}
y^{\prime}+p(x) y & =q(x), \\
y(0) & =\eta,
\end{aligned}
$$

where $p, q:[0,1[\rightarrow \mathbf{R}$ are integrable and continuous functions, and $\eta \in \mathbf{R}$. Integration is to be understood in the sense of Lebesgue. It is very well 
known that the unique solution of this problem is given by the formula

$$
y(x)=e^{-\int_{0}^{x} p(t) d t}\left(\eta+\int_{0}^{x} q(t) e^{\int_{0}^{t} p(s) d s} d t\right) \quad(0 \leqq x<1) .
$$

Our aim is to establish a procedure in order to approximate the solution by Walsh polynomials of the form

$$
\bar{y}_{n}(x)=\sum_{k=0}^{2^{n}-1} c_{k} w_{k}(x)
$$

where $w_{k}$ is the $k$ th Walsh function ordered in Paley's sense. In this regards, we discretize the equivalent integral equation

$$
y(x)=\eta+\int_{0}^{x} q(t)-p(t) y(t) d t \quad(0 \leqq x<1)
$$

replacing the functions that appear in it by the $2^{n}$-th partial sums of their Walsh-Fourier series. In other words, we find the Walsh polynomial $\bar{y}_{n}$ satisfying the relation

$$
\bar{y}_{n}(x)=\eta+S_{2^{n}}\left(\int_{0} S_{2^{n}} q(t)-S_{2^{n}} p(t) \bar{y}_{n}(t) d t\right)(x)
$$

for all $0 \leqq x<1$, where $S_{2^{n}} f$ denotes the $2^{n}$-th partial sums of Walsh-Fourier series of the integrable function $f$. We would point out that the expression

$$
S_{2^{n}}\left(\int_{0} S_{2^{n}} q(t)-S_{2^{n}} p(t) \bar{y}_{n}(t) d t\right)(x)
$$

denotes the $2^{n}$-th partial sums of Walsh-Fourier series of the integral function

$$
\int_{0}^{x} S_{2^{n}} q(t)-S_{2^{n}} p(t) \bar{y}_{n}(t) d t
$$

at the point $x$. 
The discretized integral equation (5) can be written as a linear system involving the variables $c_{0}, c_{1}, \ldots, c_{2^{n}-1}$ which are the coefficients of the Walsh polynomial $\bar{y}_{n}$. This requires the introduction of the following matrix notations. First, define the column vectors

$$
\mathbf{c}:=\left(c_{0}, c_{1}, \ldots, c_{2^{n}-1}\right)^{\top} \quad \text { and } \quad \mathbf{e}_{\mathbf{0}}:=(1,0, \ldots, 0)^{\top},
$$

or more precisely, let $\mathbf{e}_{\mathbf{0}}$ be the first unit vector of size $2^{n}$. By $\mathcal{J}$ we denote the identity matrix of size $2^{n}$. Moreover, with the Fourier coefficients of the functions $p$ and $q$ (see Section 3) we define the column vector

$$
\widehat{\mathbf{q}}:=\left(\widehat{q}_{0}, \widehat{q}_{1}, \ldots, \widehat{q}_{2^{n}-1}\right)^{\top},
$$

and the matrix

$$
P:=\left(\widehat{p}_{i \oplus j}\right)_{i, j=0}^{2^{n}-1},
$$

where $i \oplus j$ is the dyadic sum of the integers $i$ and $j$ (see Section 4). Finally, we also need the introduction of the integral functions of the Walsh-Paley functions, called triangular functions (see Section 5). These functions are denoted by $J_{k}$. With the Fourier coefficients of $J_{k}$ we construct the matrix

$$
\widehat{J}:=\left(\widehat{J}_{k, j}\right)_{k, j=0}^{2^{n}-1}
$$

which is a matrix having a very special form (see Section 5).

With the matrix notations above the discretized integral equation (5) can be written as the following linear system

$$
\left(\mathcal{J}+\widehat{J}^{\top} P\right) \mathbf{c}=\eta \mathbf{e}_{\mathbf{0}}+\widehat{J}^{\top} \widehat{\mathbf{q}} .
$$

In Section 6 we prove that this linear system is solvable and it has an unique solution, except for finite numbers of $n$. The solution of the linear system gives us the coefficients $c_{0}, c_{1}, \ldots, c_{2^{n}-1}$ of the numerical solution $\bar{y}_{n}$. In Section 7 we prove that $\bar{y}_{n}$ converges uniformly to the exact solution of the initial value problem (1) on the interval $[0,1$ [. In other words, we obtain the following result.

THEOREM 1. Let $p$ and $q$ be two continuous and integrable functions defined on the interval $\left[0,1\left[\right.\right.$. Then there exists an unique Walsh polynomial $\bar{y}_{n}$ which satisfies the discretized integral equation (5), except for finite numbers of $n$. Moreover, $\bar{y}_{n}$ converges uniformly to the solution of the initial value problem (1) on the interval $[0,1[$, if $n$ tends to infinity.

The numerical solution $\bar{y}_{n}$ may be computed without solving the linear system much more quickly by a multistep algorithm. Section 8 contains how we design this algorithm. 
The established procedure can be extended for not integrable functions $p$ and $q$. We have two possibilities to do this. One is to modify the functions $p$ and $q$ limiting their values in a neighbourhood of the point $x=1$ to be integrable. The modified initial value problem has the same exact solution outside this neighbourhood, so our method gives us a numerical solution, except in this neighbourhood. The other possibility is to use the multistep algorithm. It works with the exception of the last step, generating the numerical solution $\bar{y}_{n}$ on the interval $\left[0,1-\frac{1}{2^{n}}[\right.$. You find more details in Section 9.

\section{The $2^{n}$-th partial sums of Walsh-Fourier series}

First we introduce the concept of Walsh-Paley function. Every $n \in \mathbf{N}$ can be uniquely expressed as

$$
n=\sum_{k=0}^{\infty} n_{k} 2^{k},
$$

where $n_{k}=0$ or $n_{k}=1$ for all $k \in \mathbf{N}$. This allows us to say that the sequence $\left(n_{0}, n_{1}, \ldots\right)$ is the dyadic expansion of $n$. Similarly, the dyadic expansion $\left(x_{0}, x_{1}, \ldots\right)$ of a real number $x \in[0,1[$ is given by the sum

$$
x=\sum_{k=0}^{\infty} \frac{x_{k}}{2^{k+1}},
$$

where $x_{k}=0$ or $x_{k}=1$ for all $k \in \mathbf{N}$. This expansion is not unique if $x$ is a dyadic rational, i.e. $x$ is a number of the form $\frac{i}{2^{k}}$, where $i, k \in \mathbf{N}$ and $0 \leqq i<2^{k}$. When this situation occurs we choose the expansion terminating in zeros. Define the dyadic sum of two numbers $x, y \in[0,1[$ with expansion $\left(x_{0}, x_{1}, \ldots\right)$ and $\left(y_{0}, y_{1}, \ldots\right)$ respectively by

$$
x \dot{+} y:=\sum_{k=0}^{\infty}\left|x_{k}-y_{k}\right| 2^{-(k+1)} .
$$

Walsh functions are the finite product of functions

$$
r_{k}(x):=(-1)^{x_{k}} \quad(x \in[0,1[, k \in \mathbf{N})
$$

so-called Rademacher functions. If we sort the Walsh functions as

$$
w_{n}(x):=\prod_{k=0}^{\infty} r_{k}^{n_{k}}(x) \quad(x \in[0,1[, n \in \mathbf{N}),
$$


then we obtain the Walsh-Paley system. $w_{n}$ is called the $n$th Walsh-Paley function or in other words, the $n$th Walsh function ordered in Paley's sense. A Walsh polynomial is the finite linear combination of Walsh functions.

The Walsh-Paley system is orthonormal, i.e.

$$
\int_{0}^{1} w_{n}(x) w_{m}(x) d x= \begin{cases}1, & n=m, \\ 0, & n \neq m .\end{cases}
$$

Among other things, the orthonormality of the Walsh-Paley system ensures the fact that two Walsh polynomial are equal at every point if and only if they have the same coefficients.

In this paper we deal with real functions defined on the interval $[0,1[$. For an integrable function $f$, i.e.

$$
\int_{0}^{1}|f(x)| d x<\infty
$$

we define the Fourier coefficients and partial sums of Fourier series by

$$
\begin{aligned}
\widehat{f}_{k} & :=\int_{0}^{1} f(x) w_{k}(x) d x \quad(k \in \mathbf{N}), \\
S_{n} f(x) & :=\sum_{k=0}^{n-1} \widehat{f}_{k} w_{k}(x) \quad(n \in \mathbf{N}, x \in[0,1[) .
\end{aligned}
$$

It is important to note that the $2^{n}$-th partial sums can be written as

$$
S_{2^{n}} f(x)=2^{n} \int_{I_{n}(x)} f(y) d y
$$

where the sets

$$
I_{k}(i):=\left[\frac{i-1}{2^{k}}, \frac{i}{2^{k}}\left[\quad\left(i=1, \ldots, 2^{k}\right)\right.\right.
$$

are called dyadic intervals and $I_{n}(x)$ denotes the dyadic interval which contains the value of $x . S_{2^{n}} f$ converges to $f$ in $L^{1}$-norm for every integrable 
function $f$ (see [18, p. 142]), meaning that

$$
\lim _{n \rightarrow \infty} \int_{0}^{1}\left|S_{2^{n}} f(x)-f(x)\right| d x=0 .
$$

The integral above can be estimated by the dyadic $L^{1}$ modulus of continuity of $f$. It is a sequence which tends to zero, defined by

$$
\omega_{n}^{(1)} f:=\sup \left\{\int_{0}^{1}|f(x+h)-f(x)| d x: x \in\left[0,1\left[, 0 \leqq h<2^{-n}\right\} .\right.\right.
$$

Indeed, it is not hard to prove (see [18]), that

$$
\int_{0}^{1}\left|S_{2^{n}} f(x)-f(x)\right| d x \leqq \omega_{n}^{(1)} f .
$$

A continuous function defined on the interval $[0,1$ [ does not necessarily have to be integrable. The function

$$
f:\left[0,1\left[\rightarrow \mathbf{R}, \quad f(x)=\frac{1}{1-x}\right.\right.
$$

is a clear example of this. However, if in addition, the function has a finite limit from the left of 1 , then it is integrable, since it can be extended to a continuous function on the interval $[0,1]$. In this case the function $f$ has finite dyadic modulus of continuity, defined by

$$
\omega_{n} f:=\sup \left\{|f(x+h)-f(x)|: x \in\left[0,1\left[, 0 \leqq h<2^{-n}\right\}\right.\right.
$$

which tends to zero. It is not hard to prove (see [18]), that

$$
\left|S_{2^{n}} f(x)-f(x)\right| \leqq \omega_{n} f
$$

for all $x \in\left[0,1\left[\right.\right.$. In other words, $S_{2^{n}} f$ converges to $f$ uniformly on the interval $[0,1$ [ for every continuous function $f$ with finite limit from the left of 1. Note that there are integrable and continuous functions on the interval $[0,1$ [ which do not have finite limit from the left of 1 , as in the case of the function

$$
f:\left[0,1\left[\rightarrow \mathbf{R}, \quad f(x)=\frac{1}{\sqrt{1-x}}\right.\right.
$$


When this happens, $S_{2^{n}} f$ converges to $f$ at every point of the interval [0,1[, but not uniformly.

The following lemma is especially important in the analysis of the numerical solution that we are establishing in this paper.

Lemma 1. Suppose $f:[0,1[\rightarrow \mathbf{R}$ is constant on the dyadic intervals of length $2^{-n}$ and $x \in I_{n}(i)$ for some $i=1,2, \ldots, 2^{n}$. Then

$$
S_{2^{n}}\left(\int_{0} f(t) d t\right)(x)=\frac{1}{2^{n}} \sum_{k=1}^{i-1} f\left(\frac{k-1}{2^{n}}\right)+\frac{1}{2^{n+1}} f\left(\frac{i-1}{2^{n}}\right) .
$$

Proof. Let $\chi_{I_{n}(k)}$ be the characteristic function of the interval $I_{n}(k)$, where $k=1,2, \ldots, 2^{n}$, i.e.

$$
\chi_{I_{n}(k)}(x):= \begin{cases}1, & \frac{k-1}{2^{n}} \leqq x<\frac{k}{2^{n}} \\ 0, & \text { otherwise }\end{cases}
$$

Thus,

$$
\int_{0}^{x} \chi_{I_{n}(k)}(t) d t= \begin{cases}0, & 0 \leqq x<\frac{k-1}{2^{n}} \\ x-\frac{k-1}{2^{n}}, & \frac{k-1}{2^{n}} \leqq x<\frac{k}{2^{n}} \\ \frac{1}{2^{n}}, & \frac{k}{2^{n}} \leqq x<1\end{cases}
$$

and therefore

$$
S_{2^{n}}\left(\int_{0} \chi_{I_{n}(k)}(t) d t\right)(x)= \begin{cases}0, & 0 \leqq x<\frac{k-1}{2^{n}} \\ \frac{1}{2^{n+1}}, & \frac{k-1}{2^{n} \leqq x<\frac{k}{2^{n}}}, \\ \frac{1}{2^{n}}, & \frac{k}{2^{n}} \leqq x<1\end{cases}
$$

Since $f$ is constant on the dyadic intervals of length $2^{-n}$ we have

$$
f(x)=\sum_{k=1}^{2^{n}} f\left(\frac{k-1}{2^{n}}\right) \chi_{I_{n}(k)}(x)
$$

for all $x \in\left[0,1\left[\right.\right.$. For this reason, if $x \in I_{n}(i)$ we have

$$
S_{2^{n}}\left(\int_{0} f(t) d t\right)(x)=\sum_{k=1}^{2^{n}} f\left(\frac{k-1}{2^{n}}\right) S_{2^{n}}\left(\int_{0} \chi_{I_{n}(k)}(t) d t\right)(x)
$$




$$
=\sum_{k=1}^{i-1} f\left(\frac{k-1}{2^{n}}\right) \frac{1}{2^{n}}+f\left(\frac{i-1}{2^{n}}\right) \frac{1}{2^{n+1}}+\sum_{k=i+1}^{2^{n}} f\left(\frac{k-1}{2^{n}}\right) \cdot 0
$$

from which we obtain the statement of the lemma.

\section{Dyadically circulant matrices}

Define the dyadic sum of a pair of non-negative integers $i$ and $j$ by

$$
i \oplus j:=\sum_{k=0}^{\infty}\left|i_{k}-j_{k}\right| 2^{k}
$$

where $\left(i_{0}, i_{1}, \ldots\right)$ and $\left(j_{0}, j_{1}, \ldots\right)$ are the dyadic expansion of the integers $i$ and $j$ respectively. For all positive integer $n$ the set $\left\{0,1, \ldots, 2^{n}-1\right\}$ with the dyadic sum forms a group.

A square matrix $A$ of size $2^{n}$ is called a dyadically circulant matrix (see [13]) if all its rows can be obtained from the elements $a_{0}, a_{1}, \ldots, a_{2^{n}-1}$ in its first row by the dyadic sum, satisfying the following rule

$$
a_{i, j}=a_{i \oplus j} \quad\left(i, j=0,1, \ldots, 2^{n}-1\right)
$$

where $a_{i, j}$ is the entry in the $i$-th row and $j$-th column of $A$. In this way, the matrix has the following form

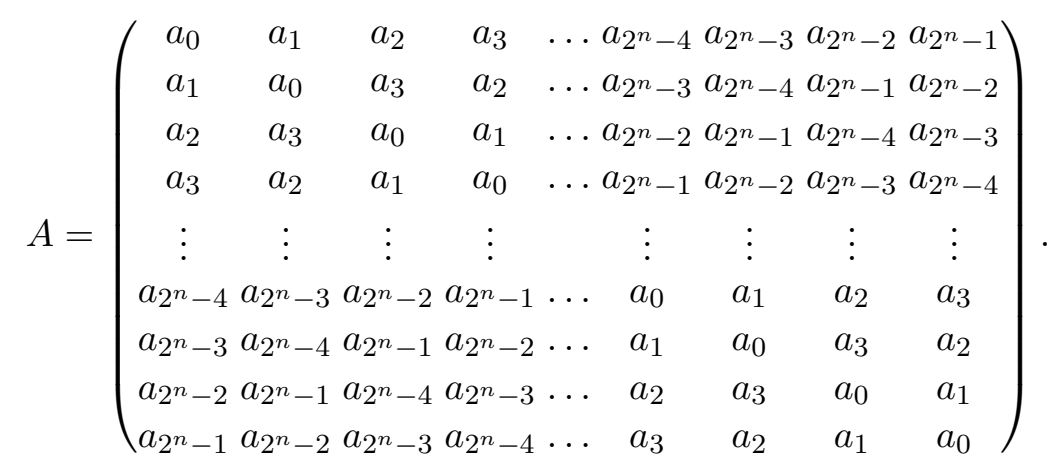

We also say that $A$ is the dyadically circulant matrix generated by the numbers $a_{0}, a_{1}, \ldots, a_{2^{n}-1}$. 
By the concept of the dyadic sum, it is not hard to see the fact that $A$ is the dyadically circulant matrix generated by the numbers $a_{0}, a_{1}, \ldots, a_{2^{n}-1}$ if and only if $A$ can be partitioned as follows

$$
A=\left(\begin{array}{c|c}
A_{0} & A_{1} \\
\hline A_{1} \mid A_{0}
\end{array}\right)
$$

where $A_{0}$ and $A_{1}$ are the dyadically circulant matrix generated by the numbers $a_{0}, a_{1}, \ldots, a_{2^{n-1}-1}$ and $a_{2^{n-1}}, a_{2^{n-1}+1}, \ldots, a_{2^{n}-1}$ respectively. This means that every dyadically circulant matrix is symmetric and therefore diagonalizable. More precisely, the dyadically circulant matrix $A$ can be written by the form

$$
A=C D C^{-1},
$$

where $D$ is a diagonal matrix made up of the eigenvalues of $A$ and $C$ is an orthogonal matrix made up of the eigenvectors of $A$. The following lemma gives us the diagonalization of dyadically circulant matrices.

Lemma 2. Let $A$ the dyadically circulant matrix generated by the numbers $a_{0}, a_{1}, \ldots, a_{2^{n}-1}$ and consider the Walsh polynomial

$$
a(x)=\sum_{j=0}^{2^{n}-1} a_{j} w_{j}(x) \quad(x \in[0,1[) .
$$

Thus, the eigenvalues of the matrix $A$ are given by the form $\lambda_{k}=a\left(\frac{k}{2^{n}}\right)$ for all $k=0,1, \ldots, 2^{n}-1$ and the eigenvector which corresponds to the eigenvalue $\lambda_{k}$ is

$$
\boldsymbol{w}_{\mathbf{k}}=\left(w_{k}(0), w_{k}\left(\frac{1}{2^{n}}\right), \ldots, w_{k}\left(\frac{2^{n}-1}{2^{n}}\right)\right)^{\top} .
$$

Proof. We prove that

$$
A \boldsymbol{w}_{\mathrm{k}}=\lambda_{k} \boldsymbol{w}_{\mathrm{k}}
$$

for all $k=0,1, \ldots, 2^{n}-1$, from which the lemma directly follows. In this regard we prove that the $i$ th element of the vectors $A \boldsymbol{w}_{\mathbf{k}}$ and $\lambda_{k} \boldsymbol{w}_{\mathbf{k}}$ are the same. Indeed

$$
\begin{aligned}
\sum_{j=0}^{2^{n}-1} a_{i, j} w_{k}\left(\frac{j}{2^{n}}\right) & =\sum_{j=0}^{2^{n}-1} a_{i \oplus j} w_{k}\left(\frac{j}{2^{n}}\right)=\sum_{r=0}^{2^{n}-1} a_{r} w_{k}\left(\frac{r \oplus i}{2^{n}}\right) \\
& =\sum_{r=0}^{2^{n}-1} a_{r} w_{k}\left(\frac{r}{2^{n}}\right) w_{k}\left(\frac{i}{2^{n}}\right)
\end{aligned}
$$




$$
\begin{aligned}
& =\sum_{r=0}^{2^{n}-1} a_{r} w_{r}\left(\frac{k}{2^{n}}\right) w_{k}\left(\frac{i}{2^{n}}\right)=a\left(\frac{k}{2^{n}}\right) w_{k}\left(\frac{i}{2^{n}}\right) \\
& =\lambda_{k} w_{k}\left(\frac{i}{2^{n}}\right) .
\end{aligned}
$$

In the computation above we use the substitution $r=i \oplus j$ and the fact that the set $\left\{0,1, \ldots, 2^{n}-1\right\}$ is a group under the dyadic sum. Moreover, we also use the elementary properties of the Walsh-Paley functions (see [18]). This completes the proof of the lemma.

The lemma above tell us that if the dyadically circulant matrix $A$ is generated by the coefficients of the Walsh polynomial

$$
a(x)=\sum_{j=0}^{2^{n}-1} a_{j} w_{j}(x) \quad(x \in[0,1[),
$$

then $A$ can be written as $A=W D_{a} W^{-1}$, where

$$
D_{a}=\left(\begin{array}{ccccc}
a(0) & 0 & 0 & \ldots & 0 \\
0 & a\left(\frac{1}{2^{n}}\right) & 0 & \ldots & 0 \\
0 & 0 & a\left(\frac{2}{2^{n}}\right) & \ldots & 0 \\
\vdots & \vdots & \vdots & \ddots & \vdots \\
0 & 0 & 0 & \ldots & a\left(\frac{2^{n}-1}{2^{n}}\right)
\end{array}\right)
$$

is a diagonal matrix and

$$
W=\left(\begin{array}{ccccc}
w_{0}(0) & w_{1}(0) & w_{2}(0) & \ldots & w_{2^{n}-1}(0) \\
w_{0}\left(\frac{1}{2^{n}}\right) & w_{1}\left(\frac{1}{2^{n}}\right) & w_{2}\left(\frac{1}{2^{n}}\right) & \ldots & w_{2^{n}-1}\left(\frac{1}{2^{n}}\right) \\
w_{0}\left(\frac{2}{2^{n}}\right) & w_{1}\left(\frac{2}{2^{n}}\right) & w_{2}\left(\frac{2}{2^{n}}\right) & \ldots & w_{2^{n}-1}\left(\frac{2}{2^{n}}\right) \\
\vdots & \vdots & \vdots & & \vdots \\
w_{0}\left(\frac{2^{n}-1}{2^{n}}\right) & w_{1}\left(\frac{2^{n}-1}{2^{n}}\right) & w_{2}\left(\frac{2^{n}-1}{2^{n}}\right) & \ldots & w_{2^{n}-1}\left(\frac{2^{n}-1}{2^{n}}\right)
\end{array}\right) .
$$

$W$ is called the Hadamard matrix of size $2^{n}$ with respect to the Walsh-Paley system. It is not hard to see that the matrix $W$ is symmetric and

$$
W^{-1}=\frac{1}{2^{n}} W
$$

This is due to the orthonormality of the Walsh-Paley system.

The following lemma is obtained directly from the diagonalization of dyadically circulant matrices and the proof is elementary linear algebra. 
Lemma 3. Let $A$ and $B$ be the dyadically circulant matrices generated by the coefficients of the Walsh polynomials

$$
a(x)=\sum_{j=0}^{2^{n}-1} a_{j} w_{j}(x) \quad \text { and } \quad b(x)=\sum_{j=0}^{2^{n}-1} b_{j} w_{j}(x)
$$

respectively, and $\alpha, \beta \in \mathbf{R}$. Then the set of dyadically circulant matrices of the same size is a commutative algebra. Moreover,

- $\alpha A+\beta B$ is the dyadically circulant matrix generated by the coefficients of the Walsh polynomial $\alpha a(x)+\beta b(x)$.

- $A B$ is the dyadically circulant matrix generated by the coefficients of the Walsh polynomial $a(x) b(x)$ (and therefore $A B=B A$ ).

- $\operatorname{det} A=\prod_{k=0}^{2^{n}-1} a\left(\frac{k}{2^{n}}\right)$.

We apply the results of Lemma 3 to calculate the determinant of a matrix related to the Fourier coefficients of triangular functions.

\section{The triangular functions}

Triangular functions are the set of integral functions of the Walsh-Paley functions. We denote them by

$$
J_{k}(x):=\int_{0}^{x} w_{k}(t) d t \quad(k \in \mathbf{N}, 0 \leqq x<1) .
$$

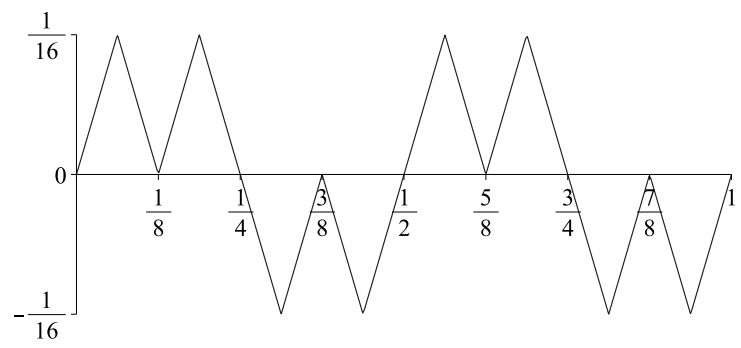

Fig. 1. The triangular function $J_{10}$ 
Let us consider the Walsh-Fourier series of the triangular functions $J_{k}$, denoting the Fourier coefficients of them by $\widehat{J}_{k, j}$, and hence

$$
J_{k}(x)=\sum_{j=0}^{\infty} \widehat{J}_{k, j} w_{j}(x) .
$$

Coefficients $\widehat{J}_{k, j}$ often take the value 0 . We can find the exact calculation of these values in [11] directly by the Fine's formulae (see [9]). With them we construct in an easier way the matrices $\widehat{J}^{(n)}$ whose entries are $\widehat{J}_{k, j}$, where $k, j=0,1, \ldots, 2^{n}-1$ :

$$
\widehat{J}^{(n)}=\left(\begin{array}{c|c|c|c}
\frac{1}{2} & \ddots & -\frac{1}{2^{n}} \mathcal{J}_{2^{n-2}} & -\frac{1}{2^{n+1}} \mathcal{J}_{2^{n-1}} \\
\hdashline \ddots & \ddots & & \\
\hline \frac{1}{2^{n}} J_{2^{n-2}} & 0_{2^{n-2}} & \\
\hline & \frac{1}{2^{n+1}} J_{2^{n-1}} & 0_{2^{n-1}}
\end{array}\right)
$$

where $\mathcal{J}_{j}$ and $0_{j}$ are the identity and null matrix of size $j$. Note that the matrix above is almost skew-symmetric, more precisely $\widehat{J}_{k, j}^{(n)}=-\widehat{J}_{j, k}^{(n)}$ if $k^{2}+$ $j^{2} \neq 0$.

For example

(8)

$$
\widehat{J}^{(3)}=\left(\begin{array}{cccccccc}
\frac{1}{2} & -\frac{1}{4} & -\frac{1}{8} & 0 & -\frac{1}{16} & 0 & 0 & 0 \\
\frac{1}{4} & 0 & 0 & -\frac{1}{8} & 0 & -\frac{1}{16} & 0 & 0 \\
\frac{1}{8} & 0 & 0 & 0 & 0 & 0 & -\frac{1}{16} & 0 \\
0 & \frac{1}{8} & 0 & 0 & 0 & 0 & 0 & -\frac{1}{16} \\
\frac{1}{16} & 0 & 0 & 0 & 0 & 0 & 0 & 0 \\
0 & \frac{1}{16} & 0 & 0 & 0 & 0 & 0 & 0 \\
0 & 0 & \frac{1}{16} & 0 & 0 & 0 & 0 & 0 \\
0 & 0 & 0 & \frac{1}{16} & 0 & 0 & 0 & 0
\end{array}\right) .
$$


Note that the matrices $\widehat{J}^{(n)}$ can be also constructed by the iteration

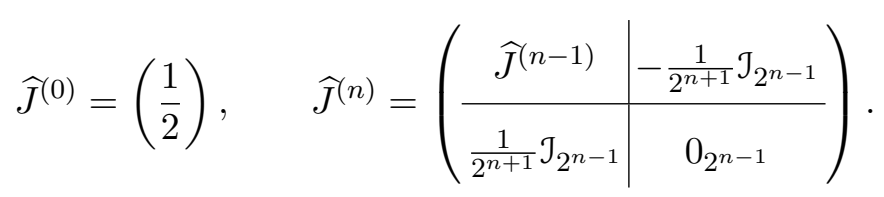

Matrices $\widehat{J}^{(n)}$ are involved in the linear system with which we obtain the numerical solution of the studied initial value problem. We will discuss it in the next section, but we must prove first an interesting relationship between $\widehat{J}^{(n)}$ and dyadically circulant matrices. In this regard we prove first the following lemma.

Lemma 4. For all positive integer $n$ we have

$$
W^{-1} \widehat{J}^{(n) \top} W=\left(\begin{array}{ccccc}
\frac{1}{2^{n+1}} & 0 & 0 & \ldots & 0 \\
\frac{1}{2^{n}} & \frac{1}{2^{n+1}} & 0 & \ldots & 0 \\
\frac{1}{2^{n}} & \frac{1}{2^{n}} & \frac{1}{2^{n+1}} & \ldots & 0 \\
\vdots & \vdots & \vdots & \ddots & \vdots \\
\frac{1}{2^{n}} & \frac{1}{2^{n}} & \frac{1}{2^{n}} & \ldots & \frac{1}{2^{n+1}}
\end{array}\right) .
$$

Proof. We compute directly the entry $a_{i j}$ of the matrix $W^{-1} \widehat{J}^{(n) \top} W$, proving that

$$
a_{i j}= \begin{cases}0, & i<j \\ \frac{1}{2^{n+1}}, & i=j \\ \frac{1}{2^{n}}, & i>j\end{cases}
$$

By the definition of the coefficients

$$
\widehat{J}_{l, k}=\int_{0}^{1} \int_{0}^{x} w_{l}(t) d t w_{k}(x) d x
$$

and by the fact that $W$ is a symmetric and orthogonal matrix such that $W^{-1}=\frac{1}{2^{n}} W$ holds, using the elementary properties of the Walsh-Paley func- 
tions (see [18]) we have

$$
\begin{aligned}
a_{i j} & =\frac{1}{2^{n}} \sum_{k=0}^{2^{n}-1} \sum_{l=0}^{2^{n}-1} w_{k}\left(\frac{i}{2^{n}}\right) \widehat{J}_{l, k} w_{l}\left(\frac{j}{2^{n}}\right) \\
& =\frac{1}{2^{n}} \sum_{k=0}^{2^{n}-1} \sum_{l=0}^{2^{n}-1} w_{k}\left(\frac{i}{2^{n}}\right) \int_{0}^{1} \int_{0}^{x} w_{l}(t) d t w_{k}(x) d x w_{l}\left(\frac{j}{2^{n}}\right) \\
& =\frac{1}{2^{n}} \sum_{k=0}^{2^{n}-1} \sum_{l=0}^{2^{n}-1} \int_{0}^{1} \int_{0}^{x} w_{l}\left(t \dot{+} \frac{j}{2^{n}}\right) d t w_{k}\left(x+\frac{i}{2^{n}}\right) d x \\
& =\frac{1}{2^{n}} \int_{0}^{1} \int_{0}^{x} D_{2^{n}}\left(t \dot{+} \frac{j}{2^{n}}\right) d t D_{2^{n}}\left(x+\frac{i}{2^{n}}\right) d x
\end{aligned}
$$

where

$$
D_{2^{n}}(x):=\sum_{k=0}^{2^{n}-1} w_{k}(x) \quad(x \in[0,1[)
$$

is called the Dirichlet kernels of order $2^{n}$. This function has the following property (see [18])

$$
D_{2^{n}}(x)= \begin{cases}2^{n}, & 0 \leqq x<\frac{1}{2^{n}} \\ 0, & \frac{1}{2^{n}} \leqq x<1\end{cases}
$$

from which we have

$$
\int_{0}^{x} D_{2^{n}}(t) d t= \begin{cases}2^{n} x, & 0 \leqq x<\frac{1}{2^{n}} \\ 1, & \frac{1}{2^{n}} \leqq x<1\end{cases}
$$

By the translation invariance of the dyadic sum and (10) we continue the calculation of the entry $a_{i j}$ as follows

$$
\begin{aligned}
a_{i j} & =\frac{1}{2^{n}} \int_{0}^{1} \int_{0}^{x+\frac{i}{2^{n}}} D_{2^{n}}\left(t+\frac{j}{2^{n}}\right) d t D_{2^{n}}(x) d x \\
& =\int_{0}^{\frac{1}{2^{n}}} \int_{0}^{x+\frac{i}{2^{n}}} D_{2^{n}}\left(t+\frac{j}{2^{n}}\right) d t d x .
\end{aligned}
$$


Note that $x+\frac{i}{2^{n}}=x+\frac{i}{2^{n}}$ if $x<\frac{1}{2^{n}}$. For this reason we can decompose the integrals above in the following way

$$
\begin{aligned}
a_{i j} & =\int_{0}^{\frac{1}{2^{n}}} \int_{0}^{x+\frac{i}{2^{n}}} D_{2^{n}}\left(t \dot{+} \frac{j}{2^{n}}\right) d t d x \\
& =\int_{0}^{\frac{1}{2^{n}}} \sum_{r=0}^{i-1} \int_{\frac{r}{2^{n}}}^{\frac{r+1}{2^{n}}} D_{2^{n}}\left(t \dot{+} \frac{j}{2^{n}}\right) d t d x+\int_{0}^{\frac{1}{2^{n}}} \int_{\frac{i}{2^{n}}}^{x+\frac{i}{2^{n}}} D_{2^{n}}\left(t \dot{+} \frac{j}{2^{n}}\right) d t d x \\
& =\int_{0}^{\frac{1}{2^{n}}} \sum_{r=0}^{i-1} \int_{\left.\frac{1}{2}^{n}+\frac{j}{2^{n}}\right)+\frac{1}{2^{n}}}^{\frac{1}{2^{n}}} D_{2^{n}}(t) d t d x+\int_{0}^{\left.\frac{i}{2^{n}}+\frac{j}{2^{n}}\right)+x} \int_{\frac{i}{2^{n}}+\frac{j}{2^{n}}} D_{2^{n}}(t) d t d x \\
& =: J_{1}+J_{2} .
\end{aligned}
$$

We obtain immediately from (10) that

$$
\int_{\frac{r}{2^{n}}+\frac{j}{2^{n}}}^{\left(\frac{r}{2^{n}}+\frac{j}{2^{n}}\right)+\frac{1}{2^{n}}} D_{2^{n}}(t) d t= \begin{cases}1, & r=j, \\ 0, & r \neq j .\end{cases}
$$

Hence

$$
J_{1}= \begin{cases}\frac{1}{2^{n}}, & i>j \\ 0, & i \leqq j\end{cases}
$$

Similarly, $J_{2}$ is not zero only if $i=j$. In this case by (11) we obtain

$$
\int_{0}^{\frac{1}{2^{n}}} \int_{\frac{i}{2^{n}}+\frac{i}{2^{n}}}^{\left(\frac{i}{2^{n}}\right.} D_{2^{n}}(t) d t d x=\int_{0}^{\left.\frac{i}{2^{n}}\right)+x} \int_{0}^{x} D_{2^{n}}(t) d t d x=\int_{0}^{\frac{1}{2^{n}}} 2^{n} x d x=\frac{1}{2^{n+1}} .
$$

Therefore,

$$
J_{2}= \begin{cases}\frac{1}{2^{n+1}}, & i=j, \\ 0, & i \neq j .\end{cases}
$$

Finally, we obtain (9) with the addition of the results obtained for $J_{1}$ and $J_{2}$, which completes the proof of the lemma. 
From the lemma above we obtain the following result.

Lemma 5. Let $A$ and $B$ be the dyadically circulant matrices generated by the coefficients of the Walsh polynomials

$$
a(x)=\sum_{j=0}^{2^{n}-1} a_{j} w_{j}(x) \quad \text { and } \quad b(x)=\sum_{j=0}^{2^{n}-1} b_{j} w_{j}(x) .
$$

respectively. Then

$$
\operatorname{det}\left(A+\widehat{J}^{(n) \top} B\right)=\prod_{i=0}^{2^{n}-1}\left(a\left(\frac{i}{2^{n}}\right)+\frac{1}{2^{n+1}} b\left(\frac{i}{2^{n}}\right)\right) .
$$

Proof. By the diagonalization of the matrices $A$ and $B$ we obtain

$$
\begin{aligned}
\operatorname{det}\left(A+\widehat{J}^{(n) \top} B\right) & =\operatorname{det}\left(W D_{a} W^{-1}+W W^{-1} \widehat{J}^{(n) \top} W D_{b} W^{-1}\right) \\
& =\operatorname{det}\left(W\left(D_{a}+W^{-1} \widehat{J}^{(n) \top} W D_{b}\right) W^{-1}\right) \\
& =\operatorname{det}\left(D_{a}+W^{-1} \widehat{J}^{(n) \top} W D_{b}\right) .
\end{aligned}
$$

Since by Lemma 4 the matrix $W^{-1} \widehat{J}^{(n) \top} W$ is triangular, then the matrix

$$
D_{a}+W^{-1} \widehat{J}^{(n) \top} W D_{b}
$$

is also triangular and the entries in the diagonal are exactly the numbers in the product of the formula which we have to prove. This means that the determinant of the matrix above is the product of these numbers, which implies that Lemma 5 holds.

\section{The existence of the numerical solution}

As we have said before, the method consists of discretizing the integral equation

$$
y(x)=\eta+\int_{0}^{x} q(t)-p(t) y(t) d t \quad(0 \leqq x<1)
$$

substituting all functions in them by the $2^{n}$ th partial sums of Walsh series of these functions. The solution $y$ is also substituted by the unknown Walsh polynomial

$$
\bar{y}_{n}(x)=\sum_{k=0}^{2^{n}-1} c_{k} w_{k}(x)
$$


Our aim is to find the Walsh polynomial $\bar{y}_{n}$ which satisfies the discretized integral equation

$$
\bar{y}_{n}(x)=\eta+S_{2^{n}}\left(\int_{0} S_{2^{n}} q(t)-S_{2^{n}} p(t) \bar{y}_{n}(t) d t\right)(x),
$$

where $w_{k}$ is the $k$ th Walsh-Paley function and operators $S_{2^{n}} f$ are the $2^{n}$-th partial sums of Walsh-Fourier series of the integrable function $f$. Remember, we suppose that $p$ and $q$ are continuous and integrable functions defined on the interval $[0,1[$.

Let us recall the matrix notation introduced in Section 2:

$$
\begin{aligned}
\mathbf{c} & :=\left(c_{0}, c_{1}, \ldots, c_{2^{n}-1}\right)^{\top}, \\
\mathbf{e}_{\mathbf{0}} & :=(1,0, \ldots, 0)^{\top} \text { with size } 2^{n}, \\
\widehat{\mathbf{q}} & :=\left(\widehat{q}_{0}, \widehat{q}_{1}, \ldots, \widehat{q}_{2^{n}-1}\right)^{\top}
\end{aligned}
$$

and

$$
\widehat{J}:=\left(\widehat{J}_{k, j}\right)_{k, j=0}^{2^{n}-1}, \quad P:=\left(\widehat{p}_{i \oplus j}\right)_{i, j=0}^{2^{n}-1} .
$$

Note that $P$ is the dyadically circulant matrix generated by the coefficients of $S_{2^{n}} p(x)$. We also introduce the notation

$$
\boldsymbol{w}(x):=\left(w_{0}(x), w_{1}(x), \ldots, w_{2^{n}-1}(x)\right)^{\top}
$$

for all $x \in[0,1[$. With these matrix notations the discretized integral equation can be written as follows

$$
\begin{aligned}
\boldsymbol{w}(x)^{\top} \mathbf{c} & =\eta+S_{2^{n}}\left(\int_{0} \boldsymbol{w}(t)^{\top} \widehat{\mathbf{q}}-\boldsymbol{w}(t)^{\top} \widehat{\mathbf{p}} \boldsymbol{w}(t)^{\top} \mathbf{c} d t\right)(x) \\
& =\eta+S_{2^{n}}\left(\int_{0} \boldsymbol{w}(t)^{\top} \widehat{\mathbf{q}}-\boldsymbol{w}(t)^{\top} P \mathbf{c} d t\right)(x) \\
& =\boldsymbol{w}(x)^{\top} \eta \mathbf{e}_{\mathbf{0}}+S_{2^{n}}\left(\int_{0} \boldsymbol{w}(t)^{\top} d t\right)(x) \cdot(\widehat{\mathbf{q}}-P \mathbf{c}) \\
& =\boldsymbol{w}(x)^{\top} \eta \mathbf{e}_{\mathbf{0}}+\boldsymbol{w}(x)^{\top} \widehat{J}^{\top}(\widehat{\mathbf{q}}-P \mathbf{c}) \\
& =\boldsymbol{w}(x)^{\top}\left(\eta \mathbf{e}_{\mathbf{0}}+\widehat{J}^{\top}(\widehat{\mathbf{q}}-P \mathbf{c})\right) .
\end{aligned}
$$


at every point of $[0,1[$. In the equation above we used the following result

$$
\begin{aligned}
\boldsymbol{w}(t)^{\top} \widehat{\mathbf{p}} \boldsymbol{w}(t)^{\top} \mathbf{c} & =\sum_{k=0}^{2^{n}-1} \widehat{p}_{k} w_{k}(t) \sum_{j=0}^{2^{n}-1} c_{j} w_{j}(t)=\sum_{k, j=0}^{2^{n}-1} \widehat{p}_{k} c_{j} w_{k}(t) w_{j}(t) \\
& =\sum_{k, j=0}^{2^{n}-1} \widehat{p}_{k} c_{j} w_{k \oplus j}(t)=\sum_{i, j=0}^{2^{n}-1} \widehat{p}_{i \oplus j} c_{j} w_{i}(t)=\boldsymbol{w}(t)^{\top} P^{\top} \mathbf{c}
\end{aligned}
$$

and in addition we use the fact that $P^{\top}=P$, since each dyadically circulant matrix is symmetric. The equality of the Walsh polynomials

$$
\boldsymbol{w}(x)^{\top} \mathbf{c}=\boldsymbol{w}(x)^{\top}\left(\eta \mathbf{e}_{\mathbf{0}}+\widehat{J}^{\top}(\widehat{\mathbf{q}}-P \mathbf{c})\right)
$$

obtained in (12) implies that they have the same coefficients, i.e.

$$
\mathbf{c}=\eta \mathbf{e}_{\mathbf{0}}+\widehat{J}^{\top}(\widehat{\mathbf{q}}-P \mathbf{c})
$$

which is a linear system involving the variables $c_{0}, c_{1}, \ldots, c_{2^{n}-1}$. This linear system can be written as follows

$$
\left(\mathcal{J}+\widehat{J}^{\top} P\right) \mathbf{c}=\eta \mathbf{e}_{\mathbf{0}}+\widehat{J}^{\top} \widehat{\mathbf{q}},
$$

where $\mathcal{J}$ is the identity matrix of size $2^{n}$.

The solvability of the linear system (13) only depends on whether the value of $\operatorname{det}\left(\mathcal{J}+\widehat{J}^{\top} P\right)$ is zero or not. Lemma 5 give us the answer, since if $A=\mathcal{J}$ and $B=P$ then we directly obtain

$$
\operatorname{det}\left(\mathcal{J}+\widehat{J}^{\top} P\right)=\prod_{i=0}^{2^{n}-1}\left(1+\frac{1}{2^{n+1}} S_{2^{n}} p\left(\frac{i}{2^{n}}\right)\right) .
$$

For this reason, the linear system (13) has an unique solution given by the formula

$$
\mathbf{c}=\left(\mathcal{J}+\widehat{J}^{\top} P\right)^{-1}\left(\eta \mathbf{e}_{\mathbf{0}}+\widehat{J}^{\top} \widehat{\mathbf{q}}\right)
$$

if $S_{2^{n}} p\left(\frac{i}{2^{n}}\right) \neq-2^{n+1}$ for all $i=0,1, \ldots, 2^{n}-1$. Otherwise, the linear system is not solvable. However, the assumption that the function $p$ is integrable means that the integral function

$$
F(x)=\int_{0}^{x} p(t) d t
$$


is absolute continuous on the closed interval $[0,1]$. From this fact it immediately follows that

$$
\lim _{n \rightarrow \infty} \max _{0 \leqq i<2^{n}}\left\{\frac{1}{2^{n}}\left|S_{2^{n}} p\left(\frac{i}{2^{n}}\right)\right|\right\}=0 .
$$

This means that $S_{2^{n}} p\left(\frac{i}{2^{n}}\right)=-2^{n+1}$ is only possible for finite numbers of $n$ and $i$. This leads us to conclude that the linear system (13) is solvable, except for a finite number of $n$. This proves the first part of Theorem 1 .

\section{The uniform convergence of the numerical solution}

In order to analyze the convergence we deal with the upper estimation of the absolute difference between the exact solution and the numerical solution of the problem (1) for every point $x \in[0,1$ [. It will be established in two steps according to

$$
\left|y(x)-\bar{y}_{n}(x)\right| \leqq\left|y(x)-S_{2^{n}} y(x)\right|+\left|S_{2^{n}} y(x)-\bar{y}_{n}(x)\right|
$$

First, let us note that by (2) the solution $y$ of the Cauchy problem (1) can be extended continuously to the closed interval $[0,1]$, since the integrability of the function $p$ and $q$ ensures that the limit

$$
\lim _{x \rightarrow 1^{-}} y(x)=e^{-\int_{0}^{1} p(t) d t}\left(\eta+\int_{0}^{1} q(t) e^{\int_{0}^{t} p(s) d s} d t\right)
$$

is finite. This means that the solution $y$ has finite modulus of continuity and

$$
\left|y(x)-S_{2^{n}} y(x)\right| \leqq \omega_{n} y
$$

for all $x \in[0,1[$ (see [18]). Therefore, the first addend of the right hand side of (16) tends uniformly to zero.

For the estimation of the second addend we introduce the function

$$
z_{n}(x):=\bar{y}_{n}(x)-S_{2^{n}} y(x) \quad(x \in[0,1[) .
$$


Thus, by (5) and (4) for all $x \in[0,1[$ we obtain

$$
\begin{aligned}
z_{n}(x)=\eta & +S_{2^{n}}\left(\int_{0} S_{2^{n}} q(t)-S_{2^{n}} p(t) \bar{y}_{n}(t) d t\right)(x) \\
& -S_{2^{n}}\left(\eta+\int_{0} q(t)-p(t) y(t) d t\right)(x) \\
= & S_{2^{n}}\left(\int_{0} S_{2^{n}} q(t)-q(t) d t\right)(x) \\
& -S_{2^{n}}\left(\int_{0}\left(S_{2^{n}} p(t)-p(t)\right) y(t) d t\right)(x) \\
& +S_{2^{n}}\left(\int_{0}^{j} S_{2^{n}} p(t)\left(y(t)-S_{2^{n}} y(t)\right) d t\right)(x) \\
& -S_{2^{n}}\left(\int_{0}^{j} S_{2^{n}} p(t) z_{n}(t) d t\right)(x) .
\end{aligned}
$$

For simplicity we use the notation

$$
\begin{aligned}
m_{n}(x):= & S_{2^{n}}\left(\int_{0} S_{2^{n}} q(t)-q(t) d t\right)(x) \\
& -S_{2^{n}}\left(\int_{0}\left(S_{2^{n}} p(t)-p(t)\right) y(t) d t\right)(x) \\
& +S_{2^{n}}\left(\int_{0} S_{2^{n}} p(t)\left(y(t)-S_{2^{n}} y(t)\right) d t\right)(x),
\end{aligned}
$$

therefore

$$
z_{n}(x)=m_{n}(x)-S_{2^{n}}\left(\int_{0} S_{2^{n}} p(t) z_{n}(t) d t\right)(x) .
$$


The functions $S_{2^{n}} p, m_{n}$ and $z_{n}$ are constants on the dyadic intervals $I_{n}(i)$ for all $i=1,2, \ldots, 2^{n}$. Hence by Lemma 1 we have

$$
\begin{aligned}
& S_{2^{n}}\left(\int_{0} S_{2^{n}} p(t) z_{n}(t) d t\right)(x) \\
& \quad=\frac{1}{2^{n}} \sum_{k=1}^{i-1} S_{2^{n}} p\left(\frac{k-1}{2^{n}}\right) z_{n}\left(\frac{k-1}{2^{n}}\right)+\frac{1}{2^{n+1}} S_{2^{n}} p\left(\frac{i-1}{2^{n}}\right) z_{n}\left(\frac{i-1}{2^{n}}\right)
\end{aligned}
$$

if $x \in I_{n}(i)$. Thus, by (18) we have

$$
\begin{aligned}
z_{n}\left(\frac{i-1}{2^{n}}\right)= & m_{n}\left(\frac{i-1}{2^{n}}\right)-\frac{1}{2^{n}} \sum_{k=1}^{i-1} S_{2^{n}} p\left(\frac{k-1}{2^{n}}\right) z_{n}\left(\frac{k-1}{2^{n}}\right) \\
& -\frac{1}{2^{n+1}} S_{2^{n}} p\left(\frac{i-1}{2^{n}}\right) z_{n}\left(\frac{i-1}{2^{n}}\right)
\end{aligned}
$$

for all $i=1,2, \ldots, 2^{n}$, which can be written as

$$
\begin{aligned}
(1 & \left.+\frac{1}{2^{n+1}} S_{2^{n}} p\left(\frac{i-1}{2^{n}}\right)\right) z_{n}\left(\frac{i-1}{2^{n}}\right) \\
& =m_{n}\left(\frac{i-1}{2^{n}}\right)-\frac{1}{2^{n}} \sum_{k=1}^{i-1} S_{2^{n}} p\left(\frac{k-1}{2^{n}}\right) z_{n}\left(\frac{k-1}{2^{n}}\right) .
\end{aligned}
$$

By (15) the sequence $\frac{1}{2^{n}} S_{2^{n}} p(x)$ tends to zero uniformly on the interval [0,1[. For this reason there exists an $n_{0} \in \mathbf{N}$ such that

$$
\left|\frac{1}{2^{n+1}} S_{2^{n}} p\left(\frac{k}{2^{n}}\right)\right|<\frac{1}{2}
$$

if $n>n_{0}$ for all $k=0,1, \ldots, 2^{n}-1$, and in this case $1+\frac{1}{2^{n+1}} S_{2^{n}} p\left(\frac{k}{2^{n}}\right) \neq 0$. Thus, we can define the numbers

$$
\rho_{k}^{(n)}:=\frac{\frac{1}{2^{n}} S_{2^{n}} p\left(\frac{k}{2^{n}}\right)}{1+\frac{1}{2^{n+1}} S_{2^{n}} p\left(\frac{k}{2^{n}}\right)} \quad\left(k=0,1, \ldots, 2^{n}-1\right) .
$$


From the formula (19) it is not difficult to prove that

$$
\begin{aligned}
(1 & \left.+\frac{1}{2^{n+1}} S_{2^{n}} p\left(\frac{i-1}{2^{n}}\right)\right) z_{n}\left(\frac{i-1}{2^{n}}\right) \\
& =m_{n}\left(\frac{i-1}{2^{n}}\right)-\sum_{k=1}^{i-1} \rho_{k-1}^{(n)} m_{n}\left(\frac{k-1}{2^{n}}\right) \prod_{j=k+1}^{i-1}\left(1-\rho_{j-1}^{(n)}\right)
\end{aligned}
$$

for all $i=1,2, \ldots, 2^{n}$. Indeed, both expressions give the same value for $i=1$, that is

$$
\left(1+\frac{1}{2^{n+1}} S_{2^{n}} p(0)\right) z_{n}(0)=m_{n}(0)
$$

and supposing that (20) holds for all numbers $1,2, \ldots, i-1$ we obtain from (19) that

$$
\begin{aligned}
(1+ & \left.\frac{1}{2^{n+1}} S_{2^{n}} p\left(\frac{i}{2^{n}}\right)\right) z_{n}\left(\frac{i}{2^{n}}\right) \\
= & m_{n}\left(\frac{i}{2^{n}}\right)-\frac{1}{2^{n}} \sum_{s=1}^{i} S_{2^{n}} p\left(\frac{s-1}{2^{n}}\right) z_{n}\left(\frac{s-1}{2^{n}}\right) \\
= & m_{n}\left(\frac{i}{2^{n}}\right)-\sum_{s=1}^{i} \frac{1}{2^{n}} S_{2^{n}} p\left(\frac{s-1}{2^{n}}\right) \frac{1}{1+\frac{1}{2^{n+1}} S_{2^{n}} p\left(\frac{s-1}{2^{n}}\right)} \\
& \times\left(m_{n}\left(\frac{s-1}{2^{n}}\right)-\sum_{k=1}^{s-1} \rho_{k-1}^{(n)} m_{n}\left(\frac{k-1}{2^{n}}\right) \prod_{j=k+1}^{s-1}\left(1-\rho_{j-1}^{(n)}\right)\right) \\
= & m_{n}\left(\frac{i}{2^{n}}\right) \\
& -\sum_{s=1}^{i} \rho_{s-1}^{(n)}\left(m_{n}\left(\frac{s-1}{2^{n}}\right)-\sum_{k=1}^{s-1} \rho_{k-1}^{(n)} m_{n}\left(\frac{k-1}{2^{n}}\right) \prod_{j=k+1}^{s-1}\left(1-\rho_{j-1}^{(n)}\right)\right) \\
= & m_{n}\left(\frac{i}{2^{n}}\right)-\sum_{s=1}^{i} \rho_{s-1}^{(n)} m_{n}\left(\frac{s-1}{2^{n}}\right) \\
& +\sum_{s=1}^{i} \sum_{k=1}^{s-1} \rho_{k-1}^{(n)} m_{n}\left(\frac{k-1}{2^{n}}\right) \prod_{j=k+1}^{s-1}\left(1-\rho_{j-1}^{(n)}\right) \rho_{s-1}^{(n)} \\
= & m_{n}\left(\frac{i}{2^{n}}\right)-\sum_{k=1}^{i} \rho_{k-1}^{(n)} m_{n}\left(\frac{k-1}{2^{n}}\right)
\end{aligned}
$$




$$
\begin{aligned}
& +\sum_{k=1}^{i} \sum_{s=k+1}^{i} \rho_{k-1}^{(n)} m_{n}\left(\frac{k-1}{2^{n}}\right) \prod_{j=k+1}^{s-1}\left(1-\rho_{j-1}^{(n)}\right) \rho_{s-1}^{(n)} \\
= & m_{n}\left(\frac{i}{2^{n}}\right)-\sum_{k=1}^{i} \rho_{k-1}^{(n)} m_{n}\left(\frac{k-1}{2^{n}}\right)\left(1-\sum_{s=k+1}^{i} \prod_{j=k+1}^{s-1}\left(1-\rho_{j-1}^{(n)}\right) \rho_{s-1}^{(n)}\right) \\
= & m_{n}\left(\frac{i}{2^{n}}\right)-\sum_{k=1}^{i} \rho_{k-1}^{(n)} m_{n}\left(\frac{k-1}{2^{n}}\right) \prod_{j=k+1}^{i}\left(1-\rho_{j-1}^{(n)}\right)
\end{aligned}
$$

which means that (20) holds for $i$. Therefore, (20) holds for all $i=$ $1,2, \ldots, 2^{n}$. In the calculations above we use the equality

$$
1-\sum_{s=k+1}^{i} \prod_{j=k+1}^{s-1}\left(1-\rho_{j-1}^{(n)}\right) \rho_{s-1}^{(n)}=\prod_{j=k+1}^{i}\left(1-\rho_{j-1}^{(n)}\right)
$$

which can be easily proved by using iteratively the transformation

$$
\begin{aligned}
1 & -\sum_{s=k+1}^{i} \prod_{j=k+1}^{s-1}\left(1-\rho_{j-1}^{(n)}\right) \rho_{s-1}^{(n)}=1-\rho_{k}^{(n)}-\sum_{s=k+2}^{i} \prod_{j=k+1}^{s-1}\left(1-\rho_{j-1}^{(n)}\right) \rho_{s-1}^{(n)} \\
& =1-\rho_{k}^{(n)}-\left(1-\rho_{k}^{(n)}\right) \sum_{s=k+2}^{i} \prod_{j=k+2}^{s-1}\left(1-\rho_{j-1}^{(n)}\right) \rho_{s-1}^{(n)} \\
& =\left(1-\rho_{k}^{(n)}\right)\left(1-\sum_{s=k+2}^{i} \prod_{j=k+2}^{s-1}\left(1-\rho_{j-1}^{(n)}\right) \rho_{s-1}^{(n)}\right) .
\end{aligned}
$$

To estimate the absolute value of $z_{n}$ we deal first with the function $m_{n}$. Note that for all $x \in[0,1[$ we have

$$
\begin{aligned}
\left|S_{2^{n}}\left(\int_{0} S_{2^{n}} q(t)-q(t) d t\right)(x)\right| & \leqq S_{2^{n}}\left(\int_{0}\left|S_{2^{n}} q(t)-q(t)\right| d t\right)(x) \\
& \leqq \int_{0}^{1}\left|S_{2^{n}} q(t)-q(t)\right| d t \leqq \omega_{n}^{(1)} q
\end{aligned}
$$


and

$$
\begin{aligned}
\left|S_{2^{n}}\left(\int_{0}\left(S_{2^{n}} p(t)-p(t)\right) y(t) d t\right)(x)\right| & \leqq S_{2^{n}}\left(\int_{0}\left|S_{2^{n}} p(t)-p(t) y(t)\right| d t\right)(x) \\
& \leqq \int_{0}^{1}\left|S_{2^{n}} p(t)-p(t) \| y(t)\right| d t \\
& \leqq\|y\|_{\infty} \omega_{n}^{(1)} p,
\end{aligned}
$$

where $\|y\|_{\infty}:=\sup \{|y(x)|: x \in[0,1[\}$ which is finite since $y$ is a bounded function on $\left[0,1\left[\right.\right.$, while $\omega_{n}^{(1)} p$ and $\omega_{n}^{(1)} q$ denote the dyadic $L^{1}$ modulus of continuity of the functions $p$ and $q$ respectively. Moreover,

$$
\int_{\frac{j-1}{2^{n}}}^{\frac{j}{2^{n}}} S_{2^{n}} y(t)-y(t) d t=0 \quad\left(j=1,2, \ldots, 2^{n}\right)
$$

and $S_{2^{n}} p$ is constant on the all dyadic intervals $I_{n}(j)$. Hence, if $x \in I_{n}(i)$ we have

$$
\begin{aligned}
S_{2^{n}} & \left(\int_{0} S_{2^{n}} p(t)\left(y(t)-S_{2^{n}} y(t)\right) d t\right)(x) \\
& =2^{n} \int_{\frac{i-1}{2^{n}}}^{\frac{i}{2^{n}}} \int_{0}^{\tau} S_{2^{n}} p(t)\left(y(t)-S_{2^{n}} y(t)\right) d t d \tau \\
& =2^{n} \int_{\frac{i-1}{2^{n}}}^{\frac{i}{2^{n}}} \int_{\frac{i-1}{2^{n}}}^{\tau} S_{2^{n}} p(t)\left(y(t)-S_{2^{n}} y(t)\right) d t d \tau \\
& =2^{n} S_{2^{n}} p(x) \int_{\frac{i-1}{2^{n}}}^{\frac{i}{2^{n}}} \int_{\frac{i-1}{2^{n}}}^{\tau} y(t)-S_{2^{n}} y(t) d t d \tau
\end{aligned}
$$


and then

$$
\begin{aligned}
& \left|S_{2^{n}}\left(\int_{0} S_{2^{n}} p(t)\left(y(t)-S_{2^{n}} y(t)\right) d t\right)(x)\right| \\
& \quad \leqq 2^{n}\left|S_{2^{n}} p(x)\right| \int_{\frac{i-1}{2^{n}}}^{\frac{i}{2^{n}}} \int_{\frac{i-1}{2^{n}}}^{\tau}\left|y(t)-S_{2^{n}} y(t)\right| d t d \tau \\
& \quad \leqq 2^{n}\left|S_{2^{n}} p(x)\right| \int_{2^{n}}^{\frac{i}{2^{n}}} \int_{\frac{i-1}{2^{n}}}^{\frac{i-1}{2^{n}}}\left|y(t)-S_{2^{n}} y(t)\right| d t d \tau \\
& \quad \leqq \max _{0 \leq 2^{n}}\left\{\frac{1}{2^{n}}\left|S_{2^{n}} p\left(\frac{i}{2^{n}}\right)\right|\right\} \omega_{n} y .
\end{aligned}
$$

Summarizing our results we have that $\left|m_{n}(x)\right| \leqq M_{n}$ for all $x \in[0,1[$, where

$$
M_{n}:=\omega_{n}^{(1)} q+\|y\|_{\infty} \omega_{n}^{(1)} p+\max _{0 \leqq i<2^{n}}\left\{\frac{1}{2^{n}}\left|S_{2^{n}} p\left(\frac{i}{2^{n}}\right)\right|\right\} \omega_{n} y
$$

which by (15) tends to zero if $n \rightarrow \infty$. Thus, by (20) we obtain

$$
\begin{aligned}
\mid 1 & +\frac{1}{2^{n+1}} S_{2^{n}} p\left(\frac{i-1}{2^{n}}\right)|| z_{n}\left(\frac{i-1}{2^{n}}\right) \mid \\
& \leqq M_{n}+\sum_{k=1}^{i-1}\left|\rho_{k-1}^{(n)}\right| M_{n} \prod_{j=k+1}^{i-1}\left(1+\left|\rho_{j-1}^{(n)}\right|\right) \\
& =M_{n}\left(1+\sum_{k=1}^{i-1}\left|\rho_{k-1}^{(n)}\right| \prod_{j=k+1}^{i-1}\left(1+\left|\rho_{j-1}^{(n)}\right|\right)\right) \\
& =M_{n} \prod_{k=1}^{i-1}\left(1+\left|\rho_{k-1}^{(n)}\right|\right) .
\end{aligned}
$$

In the calculations above we use the equality

$$
1+\sum_{k=1}^{i-1}\left|\rho_{k-1}^{(n)}\right| \prod_{j=k+1}^{i-1}\left(1+\left|\rho_{j-1}^{(n)}\right|\right)=\prod_{k=1}^{i-1}\left(1+\left|\rho_{k-1}^{(n)}\right|\right)
$$


which can be easily proved by using iteratively the transformation

$$
\begin{aligned}
1 & +\sum_{k=1}^{i-1}\left|\rho_{k-1}^{(n)}\right| \prod_{j=k+1}^{i-1}\left(1+\left|\rho_{j-1}^{(n)}\right|\right)=1+\left|\rho_{i-2}^{(n)}\right|+\sum_{k=1}^{i-2}\left|\rho_{k-1}^{(n)}\right| \prod_{j=k+1}^{i-1}\left(1+\left|\rho_{j-1}^{(n)}\right|\right) \\
& =1+\left|\rho_{i-2}^{(n)}\right|+\left(1+\left|\rho_{i-2}^{(n)}\right|\right) \sum_{k=1}^{i-2}\left|\rho_{k-1}^{(n)}\right| \prod_{j=k+1}^{i-2}\left(1+\left|\rho_{j-1}^{(n)}\right|\right) \\
& =\left(1+\left|\rho_{i-2}^{(n)}\right|\right)\left(1+\sum_{k=1}^{i-2}\left|\rho_{k-1}^{(n)}\right| \prod_{j=k+1}^{i-2}\left(1+\left|\rho_{j-1}^{(n)}\right|\right)\right) .
\end{aligned}
$$

We know that

$$
\left|\frac{1}{2^{n+1}} S_{2^{n}} p\left(\frac{k}{2^{n}}\right)\right|<\frac{1}{2}
$$

for all $k=0,1, \ldots, 2^{n}-1$, if $n>n_{0}$. Hence,

$$
1+\frac{1}{2^{n+1}} S_{2^{n}} p\left(\frac{i-1}{2^{n}}\right)>\frac{1}{2}, \quad\left|\rho_{k}^{(n)}\right|<\frac{1}{2^{n-1}}\left|S_{2^{n}} p\left(\frac{k}{2^{n}}\right)\right| .
$$

Therefore,

$$
\begin{aligned}
\frac{1}{2}\left|z_{n}\left(\frac{i-1}{2^{n}}\right)\right| & \leqq M_{n} \prod_{k=1}^{i-1}\left(1+\frac{1}{2^{n-1}}\left|S_{2^{n}} p\left(\frac{k-1}{2^{n}}\right)\right|\right) \\
& =M_{n} \prod_{k=1}^{i-1}\left(1+2\left|\int_{\frac{k-1}{2^{n}}}^{\frac{k}{2^{n}}} p(x) d x\right|\right) \\
& \leqq M_{n} \prod_{k=1}^{i-1}\left(1+2 \int_{\frac{k-1}{2^{n}}}^{\frac{k}{2^{n}}}|p(x)| d x\right)
\end{aligned}
$$


if $n>n_{0}$. Thus, for $i=1$ we have $\left|z_{n}(0)\right| \leqq 2 M_{n}$ and by the inequality of arithmetic and geometric means we obtain

$$
\begin{aligned}
\left|z_{n}\left(\frac{i-1}{2^{n}}\right)\right| & \leqq 2 M_{n}\left(\frac{1}{i-1} \sum_{k=1}^{i-1}\left(1+2 \int_{\frac{k-1}{2^{n}}}^{\frac{k}{2^{n}}}|p(x)| d x\right)\right)^{i-1} \\
& =2 M_{n}\left(1+\frac{2}{i-1} \int_{0}^{\frac{i-1}{2^{n}}}|p(x)| d x\right)^{i-1} \\
& \leqq 2 M_{n}\left(1+\frac{2}{i-1} \int_{0}^{1}|p(x)| d x\right)^{i-1} \\
& <2 M_{n} e^{2 \int_{0}^{1}|p(x)| d x}
\end{aligned}
$$

for all $i=2,3, \ldots, 2^{n}$. Therefore,

$$
\left|z_{n}(x)\right| \leqq 2 M_{n} e^{2 \int_{0}^{1}|p(x)| d x} \quad(x \in[0,1[),
$$

which tends to zero as $n \rightarrow \infty$, meaning that the second part of (16) also tends uniformly to zero. Thus, the absolute difference

$$
\left|y(x)-\bar{y}_{n}(x)\right|
$$

tends uniformly to zero, hence the second part of Theorem 1 is true.

\section{A multistep algorithm for the numerical solution}

The disadvantage of our method is the requirement for the solution of a liner system with a very large number of equations. In addition, we must construct a Walsh polynomial with the coefficient that appear in the solution. The amount of time required for these computations would really be large if we try to obtain a high accuracy. In this section we propose a faster method for directly getting the values of the numerical solution without needing to solve the linear systems and generate Walsh polynomials. The method is based on the fact that the numerical solution $\bar{y}_{n}$ is constant on the dyadic intervals of length $2^{-n}$, i.e.

$$
\bar{y}_{n}(x)=\bar{y}_{n}\left(\frac{i-1}{2^{n}}\right) \quad\left(x \in I_{n}(i)\right)
$$


for all $i=1,2, \ldots, 2^{n}$. The point is to calculate the value of $\bar{y}_{n}\left(\frac{i-1}{2^{n}}\right)$ from the previous values $\bar{y}_{n}\left(\frac{k}{2^{n}}\right)$, where $k=0,1, \ldots, i-2$, starting from the value of $\bar{y}_{n}(0)$. It is called a multistep algorithm and this kind of algorithms are frequently used to solve differential equations numerically.

In order to design the algorithm note that the function $S_{2^{n}} q(x)-$ $S_{2^{n}} p(x) \bar{y}_{n}(x)$ is constant on the dyadic intervals of length $2^{-n}$. Thus, by (5) and Lemma 1 we obtain

$$
\begin{aligned}
\bar{y}_{n}(x)=\eta & +S_{2^{n}}\left(\int_{0} S_{2^{n}} q(t)-S_{2^{n}} p(t) \bar{y}_{n}(t) d t\right)(x) \\
=\eta & +\frac{1}{2^{n}} \sum_{k=1}^{i-1}\left(S_{2^{n}} q\left(\frac{k-1}{2^{n}}\right)-S_{2^{n}} p\left(\frac{k-1}{2^{n}}\right) \bar{y}_{n}\left(\frac{k-1}{2^{n}}\right)\right) \\
& +\frac{1}{2^{n+1}}\left(S_{2^{n}} q\left(\frac{i-1}{2^{n}}\right)-S_{2^{n}} p\left(\frac{i-1}{2^{n}}\right) \bar{y}_{n}\left(\frac{i-1}{2^{n}}\right)\right)
\end{aligned}
$$

if $x \in I_{n}(i)$ for all $i=1,2, \ldots, 2^{n}$. Note also that $\bar{y}_{n}(x)=\bar{y}_{n}\left(\frac{i-1}{2^{n}}\right)$. After solving the formula above for $\bar{y}_{n}\left(\frac{i-1}{2^{n}}\right)$ we obtain the multistep algorithm

$$
\begin{aligned}
& \bar{y}_{n}\left(\frac{i-1}{2^{n}}\right) \\
= & \frac{1}{1+\frac{1}{2^{n+1}} S_{2^{n}} p\left(\frac{i-1}{2^{n}}\right)}\left(\eta+\frac{1}{2^{n}} \sum_{k=1}^{i-1}\left(S_{2^{n}} q\left(\frac{k-1}{2^{n}}\right)-S_{2^{n}} p\left(\frac{k-1}{2^{n}}\right) \bar{y}_{n}\left(\frac{k-1}{2^{n}}\right)\right)\right. \\
& \left.+\frac{1}{2^{n+1}} S_{2^{n}} q\left(\frac{i-1}{2^{n}}\right)\right)
\end{aligned}
$$

which starts from $i=1$ taking the value

$$
\bar{y}_{n}(0)=\frac{1}{1+\frac{1}{2^{n+1}} S_{2^{n}} p(0)}\left(\eta+\frac{1}{2^{n+1}} S_{2^{n}} q(0)\right) .
$$

Observe that for the multistep algorithm we only need the $2^{n}$ th partial sums of Walsh series of the functions $p$ and $q$ which are just integral means. Hence they are more simple to compute than the Walsh-Fourier coefficients that appear in the linear system. Note also that unlike others multistep algorithms the value of $\bar{y}_{n}(0)$ does not necessarily have to be $\eta$, but it tends to $\eta$ if $n \rightarrow \infty$. 


\section{The extension of the method for not integrable functions}

The assumption of integrability for the functions $p$ and $q$ is essential to implement our method, since the Walsh-Fourier coefficients of $p$ and $q$ are needed for the linear system, on the other hand, the partial sums $S_{2^{n}} p$ and $S_{2^{n}} q$ are required for the multistep algorithm. What we may do in case that $p$ and $q$ are not integrable, but continuous functions on the interval $[0,1[$ ?

In this regard consider the initial value problem

$$
\begin{aligned}
y^{\prime}+p(x) y & =q(x), \\
y(0) & =\eta,
\end{aligned}
$$

where $p, q:[0,1[\rightarrow \mathbf{R}$ are continuous functions and $\eta \in \mathbf{R}$, but $p$ or $q$ is not integrable on $[0,1]$. The situation is quite different now, since the solution $y$ would be unbounded. In this case a numerical solution formed by Walsh polynomials can not converge to $y$. But the continuity ensures that for all $0<\alpha<1$ numbers $p$ and $q$ are integrable on $[0, \alpha]$. For this reason, we propose to modify the initial value problem (21) as follows. Let $0<\alpha<1$ be a fixed number, and define

$$
p^{*}(x):=\left\{\begin{array}{ll}
p(x), & 0 \leqq x<\alpha, \\
p(\alpha), & \alpha \leqq x<1,
\end{array} \quad \text { and } \quad q^{*}(x):= \begin{cases}q(x), & 0 \leqq x<\alpha \\
q(\alpha), & \alpha \leqq x<1\end{cases}\right.
$$

Now consider the modified initial value problem

$$
\begin{aligned}
y^{\prime}+p^{*}(x) y & =q^{*}(x), \\
y(0) & =\eta .
\end{aligned}
$$

Note that the functions $p^{*}$ and $q^{*}$ are continuous and integrable, hence Theorem 1 is valid for the modified problem above. The uniqueness of the solution of a general initial value problems for linear differential equations implies that the original and the modified initial problem have the same solution on the interval $[0, \alpha[$. Therefore, the procedure implemented for the modified problem gives us a numerical solution $\bar{y}_{n}$ which converges uniformly to the exact solution of the original problem on the interval $[0, \alpha[$. Consequently, if we like to obtain an approximation of the solution at a point $x \in[0,1$ [ we may take a value of $\alpha$ greater than $x$ and numerically solve the modified initial value problem by our method. 
However, in practice it is better to use the multistep algorithm. It is usable since the continuity of the functions $p$ and $q$ ensures that the integrals

$$
S_{2^{n}} p\left(\frac{i-1}{2^{n}}\right)=2^{n} \int_{\frac{i-1}{2^{n}}}^{\frac{i}{2^{n}}} p(t) d t, \quad S_{2^{n}} q\left(\frac{i-1}{2^{n}}\right)=2^{n} \int_{\frac{i-1}{2^{n}}}^{\frac{i}{2^{n}}} q(t) d t
$$

exist for all $i=1,2, \ldots, 2^{n}-1$. For $i=2^{n}$ the integrals above may be calculated only if the functions $p$ and $q$ are integrable on the whole interval $[0,1]$. This means that the multistep algorithm can always be implemented, except the last step.

In case that $\alpha=1-\frac{1}{2^{n}}$ we have

$$
S_{2^{n}} p^{*}\left(\frac{i-1}{2^{n}}\right)=S_{2^{n}} p\left(\frac{i-1}{2^{n}}\right) \quad \text { and } \quad S_{2^{n}} q^{*}\left(\frac{i-1}{2^{n}}\right)=S_{2^{n}} q\left(\frac{i-1}{2^{n}}\right)
$$

for all $1 \leqq i \leqq 2^{n}-1$. Consequently, the multistep algorithm generates the same solution for the original and the modified initial problem on the interval $\left[0,1-\frac{1}{2^{n}}\left[\right.\right.$. The remaining part, i.e. the interval $\left[1-\frac{1}{2^{n}}, 1\left[\right.\right.$, is of length $\frac{1}{2^{n}}$ which tends to zero if $n$ tends to infinity. This allows us to say, that the numerical solution $\bar{y}_{n}$ generated by the multistep algorithm converges to the exact solution $\bar{y}$ of the original problem at every point of the interval $[0,1[$. We mean that for every $x \in\left[0,1\left[\right.\right.$ we can find an $n_{0}$ positive integer such that $x$ is in the domain of $\bar{y}_{n}$ for all $n \geqq n_{0}$ and $\bar{y}_{n}(x) \rightarrow y(x)$. The convergence is uniform on all closed subinterval of $[0,1[$.

\section{Examples}

In the first instance, consider the problem

$$
\begin{aligned}
y^{\prime}+\tan (x) y & =\sin 2 x, \\
y(0) & =2 .
\end{aligned}
$$

Note that the functions $p(x)=\tan x$ and $q(x)=\sin 2 x$ are both continuous on the interval $[0,1]$, therefore they are integrable with a finite limit from the left of 1 . In this case the numerical solutions $\bar{y}_{n}$ converge uniformly to the exact solution of the problem which is

$$
y(x)=4 \cos x-2 \cos ^{2} x .
$$

Figure 2 illustrates how close is the numerical solution $\bar{y}_{4}$ to the solution $y$. Moreover, Table 1 show us how fast the convergence is. We can see that the 
supremum of the absolute difference between the solution and the numerical solution is reduced almost by half if the value of $n$ increases by one. We made all computations in this section by Maple with the multistep algorithm.

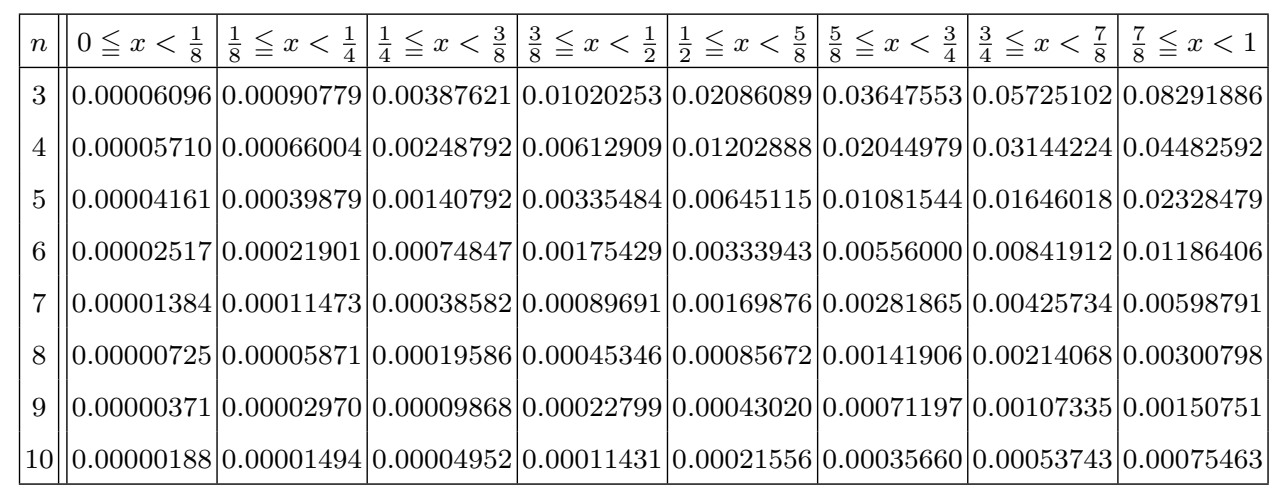

Table 1. sup $\left|y(x)-\bar{y}_{n}(x)\right|$ on the dyadic intervals of length $\frac{1}{8}$ for (23)

The second example shows us a problem with integrable functions $p$ and $q$, but one of them does not have a finite limit from the left of 1 . Consider the problem

$$
\begin{aligned}
y^{\prime}+\frac{3}{2 \sqrt{1-x}} y & =1-x-\sqrt{1-x}, \\
y(0) & =\frac{2}{3} .
\end{aligned}
$$

In this case

$$
p(x)=\frac{3}{2 \sqrt{1-x}} \quad \text { and } \quad q(x)=1-x-\sqrt{1-x}
$$

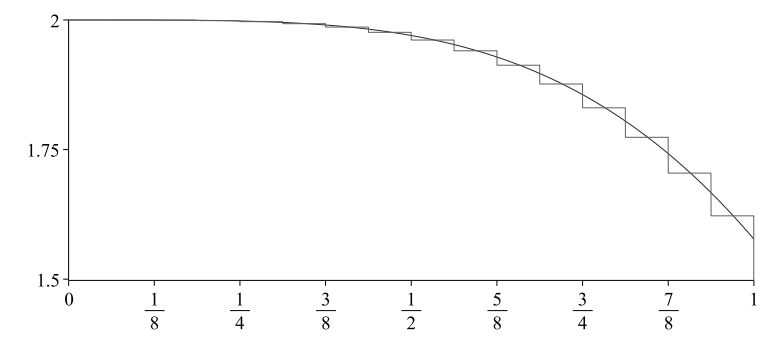

Fig. 2. $\bar{y}_{4}$, the numerical solution of the problem (23) for $n=4$ 
which are both integrable even though the limit from the left of 1 of the function $p$ is infinite. Theorem 1 is also valid in this case, therefore the numerical solutions $\bar{y}_{n}$ also converge uniformly to the exact solution of the problem which is

$$
y(x)=\frac{2}{3} \sqrt{(1-x)^{3}}
$$

The behavior of the convergence is similar to the previous problem. We can also see in the following Table 2 that the supremum of the absolute difference between the solution and the numerical solution is reduced almost by half if the value of $n$ increases by one.

\begin{tabular}{|l||l|l|l|l|l|l|l|l|}
\hline$n$ & $0 \leqq x<\frac{1}{8}$ & $\frac{1}{8} \leqq x<\frac{1}{4}$ & $\frac{1}{4} \leqq x<\frac{3}{8}$ & $\frac{3}{8} \leqq x<\frac{1}{2}$ & $\frac{1}{2} \leqq x<\frac{5}{8}$ & $\frac{5}{8} \leqq x<\frac{3}{4}$ & $\frac{3}{4} \leqq x<\frac{7}{8}$ & $\frac{7}{8} \leqq x<1$ \\
\hline 3 & 0.06062299 & 0.05667439 & 0.05238233 & 0.04765793 & 0.04235894 & 0.03623165 & 0.02872980 & 0.01863694 \\
4 & 0.03077155 & 0.02877700 & 0.02662168 & 0.02426382 & 0.02163804 & 0.01863124 & 0.01501273 & 0.01011927 \\
5 & 0.01550418 & 0.01450120 & 0.01342051 & 0.01224183 & 0.01093372 & 0.00944255 & 0.00766154 & 0.00530135 \\
6 & 0.00778214 & 0.00727914 & 0.00673795 & 0.00614856 & 0.00549556 & 0.00475279 & 0.00386876 & 0.00270748 \\
7 & 0.00389864 & 0.00364675 & 0.00337593 & 0.00308121 & 0.00275496 & 0.00238425 & 0.00194378 & 0.00136753 \\
8 & 0.00195122 & 0.00182518 & 0.00168971 & 0.00154234 & 0.00137927 & 0.00119408 & 0.00097423 & 0.00068717 \\
9 & 0.00097609 & 0.00091304 & 0.00084529 & 0.00077161 & 0.00069009 & 0.00059753 & 0.00048770 & 0.00034443 \\
10 & 0.00048816 & 0.00045663 & 0.00042275 & 0.00038591 & 0.00034515 & 0.00029889 & 0.00024400 & 0.00017242 \\
\hline
\end{tabular}

Table 2. $\sup \left|y(x)-\bar{y}_{n}(x)\right|$ on the dyadic intervals of length $\frac{1}{8}$ for (24)

Finally we deal with the case that the functions $p$ and $q$ are not integrable. Consider the problem

$$
\begin{aligned}
y^{\prime}+\frac{5}{1-x} y & =\frac{5 x^{4}}{1-x}, \\
y(0) & =0 .
\end{aligned}
$$

which has the solution

$$
y(x)=x^{5}
$$

The coefficient and free term are

$$
p(x)=\frac{5}{1-x} \quad \text { and } \quad q(x)=\frac{5 x^{4}}{1-x} .
$$

Since $p$ and $q$ are not integrable functions on the interval $[0,1]$, then Theorem 1 is not valid, but we may obtain a numerical solution $\bar{y}_{n}$ on the interval $\left[0,1-\frac{1}{2^{n}}[\right.$ by the multistep algorithm. In this case we illustrate the convergence choosing some point of the interval [0,1[ and computing the absolute 
difference between the solution and the numerical solution at these points. The results appear in Table 3.

\begin{tabular}{|c||c|c|c|c|c|c|c|c|}
\hline$n$ & $x=\frac{1}{2}$ & $x=\frac{3}{4}$ & $x=\frac{7}{8}$ & $x=\frac{15}{16}$ & $x=\frac{31}{32}$ & $x=\frac{63}{64}$ & $x=\frac{127}{128}$ & $x=\frac{255}{256}$ \\
\hline 3 & 0.02993002 & 0.13766372 & - & - & - & - & - & - \\
4 & 0.01208584 & 0.05776304 & 0.11082398 & - & - & - & - & - \\
5 & 0.00543147 & 0.02667540 & 0.04993559 & 0.06924819 & - & - & - & - \\
6 & 0.00257483 & 0.01283496 & 0.02387420 & 0.03206557 & 0.03856926 & - & - & - \\
7 & 0.00125360 & 0.00629703 & 0.01168669 & 0.01553123 & 0.01807579 & 0.02033629 & - & - \\
8 & 0.00061852 & 0.00311901 & 0.00578315 & 0.00765158 & 0.00880501 & 0.00958484 & 0.01043952 & - \\
9 & 0.00030721 & 0.00155221 & 0.00287681 & 0.00379847 & 0.00434996 & 0.00468136 & 0.00493385 & 0.00528868 \\
10 & 0.00015310 & 0.00077429 & 0.00143474 & 0.00189254 & 0.00216243 & 0.00231575 & 0.00241286 & 0.00250288 \\
\hline
\end{tabular}

Table 3. Value of $\left|y(x)-\bar{y}_{n}(x)\right|$ in some points for (25)

Observe that in this case, the method only works for large values of $n$ if $x$ is close to 1 . On the other hand, we can see that in this example the absolute difference between the solution and the numerical solution is also reduced almost by half if the value of $n$ increases by one.

\section{Conclusion}

The method designed by Chen and Hsiao for solving systems of linear differential equations with constant coefficients may be extended in case of one equations with not necessarily constant coefficients. The proposed numerical solution is a Walsh polynomial, i.e. a piecewise constant function on intervals of length $\frac{1}{2^{n}}$. The approach to the problem was as general as possible, considering the function in the differential equation continuous and integrable on the interval [0,1[. In this case the numerical solution always exits, except for finite positive integers $n$, and it converges uniformly to the exact solution. The disadvantage of the method by needing to solve very large linear systems may be avoided by the use of a multistep algorithm.

With a few modifications the method also works in case that the function in the differential equation are continuous, but not integrable on the interval $[0,1[$. The multistep algorithm gives us a numerical solution on the interval $\left[0,1-\frac{1}{2^{n}}[\right.$ in this instance. This allows us to approximate the value of the exact solution at every point of the interval $[0,1[$, but the uniform convergence is not true in general.

Acknowledgement. The first author is supported by the projects EFOP3.6.1-16-2016-00022 and EFOP-3.6.2-16-2017-00015 supported by the Euro- 
pean Union, co-financed by the European Social Fund. The second author is supported by the project GINOP-2.2.1-15-2017-00055.

\section{REFERENCES}

[1] Blachman, N. M., Sinusoids versus Walsh functions, Proceedings of the IEEE, 62(3) (1974), 346-354.

[2] Chen, C. F. and Hsiao, C. H., A state-space approach to Walsh series solution of linear systems, International Journal of Systems Science, 6(9) (1975), 833858.

[3] Chen, C. F. and Hsiao, C. H., A Walsh series direct method for solving variational problems. Journal of the Franklin Institute, 300(4) (1975), 265-280.

[4] Chen, C. F. and Hsiao, C. H., Design of piecewise constant gains for optimal control via Walsh functions, IEEE Transactions on Automatic Control, 20(5) (1975), 596-603.

[5] Chen, C. F. and Hsiao, C. H., Time-domain synthesis via walsh functions, in: Proceedings of the Institution of Electrical Engineers, volume 122, pages 565570. IET, 1975.

[6] Chen, C. F. and Hsiao, C. H., Walsh series analysis in optimal control, International Journal of Control, 21(6) (1975), 881-897.

[7] Chen, W.-L. and Shin, Y.-P., Shift walsh matrix and delay-differential equations, IEEE Transactions on Automatic Control, 23(6) (1987), 1023-1028.

[8] Corrington, M., Solution of differential and integral equations with Walsh functions, IEEE Transactions on Circuit Theory, 20(5) (1973), 470-476.

[9] Fine, N. J., On the Walsh functions, Trans. Am. Math. Soc., 65 (1949), 372-414.

[10] Gát, Gy. and Toledo, T., A numerical method for solving linear differential equations via Walsh functions, in: Advances in Information Science and Applications, volume 2, pages 334-339. Proceedings of the 18th International Conference on Computers (part of CSCC 2014), Santorini Island, Greece, July 17-21, 2014, 2014.

[11] GÁt, Gy. and Toledo, R., Estimating the error of the numerical solution of linear differential equations with constant coefficients via Walsh polynomials, Acta Math. Acad. Paedagog. Nyházi. (N.S.), 31(2) (2015), 309-330.

[12] Gibbs, J. E. and Gebbie, H. A., Application of Walsh Functions to Transform Spectroscopy, Nature, 224 (1969), 1012-1013.

[13] Gulamhusein, M. N., Simple matrix-theory proof of the discrete dyadic convolution theorem, Electronics Letters, 9(10) (1973), 238-239.

[14] Harmuth, H. F., Transmission of information by orthogonal functions, 2nd ed., Berlin-Heidelberg-New York: Springer-Verlag. XII, 393 p., 1972.

[15] Lukomskit, D. S., Lukomskis, S. F. and Terekhin, P. A., Solution of Cauchy problem for equation first order via Haar functions, Izv. Saratov Univ. (N.S.), Ser. Math. Mech. Inform., 16 (2016), 151-159.

[16] Онта, T., Expansion of Walsh Functions in Terms of Shifted Rademacher Functions and Its Applications to the Signal Processing and the Radiation of Electromagnetic Walsh Waves, IEEE Transactions on Electromagnetic Compatibility, EMC-18 (1976), 201-205.

[17] RaO, G. P., Piecewise constant orthogonal functions and their application to systems and control., volume 55, Springer, Cham, 1983. 
[18] Schipp, F., Wade, W. R. and Simon, P., Walsh series. An introduction to dyadic harmonic analysis, Adam Hilger, Bristol and New York, 1990.

[19] Shin, Y.-P. and Han, J,-Y., Double walsh series solution of first-order partial differential equations, International Journal of Systems Science, 9(5) (1978), $569-578$.

[20] Stankovic, R. S. and Miller, D. M., Using QMDD in numerical methods for solving linear differential equations via Walsh functions, in: 2015 IEEE INTernational Symposium on Multiple-Valued Logic, pages 182-188, May 2015.

[21] Walsh, J. L., A closed set of normal orthogonal functions, Am. J. Math., 45 (1923), $5-24$.

Open Access statement. This is an open-access article distributed under the terms of the Creative Commons Attribution 4.0 International License (https://creativecommons.org/ licenses/by/4.0/), which permits unrestricted use, distribution, and reproduction in any medium, provided the original author and source are credited, a link to the CC License is provided, and changes âĂŞ if any âĂ as are indicated. (SID_1) 